\title{
miR-1291 targets mucin 1 inhibiting cell proliferation and invasion to promote cell apoptosis in esophageal squamous cell carcinoma
}

\author{
HAILAN LUO ${ }^{1}$, WENTAO GUO ${ }^{1}$, FEI WANG ${ }^{1}$, YANJIE YOU ${ }^{1}$, JIANGUO WANG ${ }^{1}$, XUDONG CHEN $^{1}$, \\ JIHONG WANG ${ }^{1}$, YUANYUAN WANG ${ }^{2}$, YUWEN DU ${ }^{2}$, XIAONAN CHEN ${ }^{2}$, \\ CHANGGUI XUE ${ }^{2}$, GUOHUA SONG ${ }^{1}$ and FUQING WANG ${ }^{1}$ \\ ${ }^{1}$ Department of Basic Medical Sciences, Luohe Medical College, Luohe, Henan 462002; \\ ${ }^{2}$ College of Basic Medical Sciences, Zhengzhou University, Zhengzhou, Henan 450001, P.R. China
}

Received April 30, 2015; Accepted July 10, 2015

DOI: 10.3892/or.2015.4206

\begin{abstract}
MicroRNAs (miRNAs) are well known as important regulators in various cancer development. In the present study, we focused on the expression and biological function of miR-1291 in esophageal squamous cell carcinoma (ESCC). Compared with adjacent non-tumorous tissue samples, qRT-PCR data showed significant downregulation of miR-1291 in 54 ESCC tissue samples $(\mathrm{P}<0.05)$, which was also significantly associated with lymph node metastases and clinical stage $(\mathrm{P}<0.05)$. Cell Counting Kit- $8(\mathrm{CCK}-8)$, colony formation, Transwell and flow cytometric apoptosis assays were performed to detect the effect of miR-1291 upregulation, and the results showed inhibition of the proliferation, invasion and promotion of apoptosis in EC9706 and EC-1 cells. Using bioinformatic analyses, we found that mucin 1 (MUC1) was a potential target for miR-1291. Luciferase assays were performed to reveal that miR-1291 inhibited MUC1 expression by targeting the seed region of MUC1 3'-untranslated region (3'UTR). We also found that the expression of MUC1 lacking in 3'UTR abrogated the anti-invasion and pro-apoptosis function of miR-1291. Our results demonstrated the importance of miR-1291 in targeting MUC1 for the regulation of esophagus cancer growth, invasion and apoptosis, and may be helpful for developing new targets for early diagnosis or new therapeutic targets for ESCC.
\end{abstract}

\section{Introduction}

Esophageal squamous cell carcinoma (ESCC) is a common malignancy worldwide (1). As a result of lymph node metas-

Correspondence to: Professor FuQing Wang or Professor GuoHua Song, Department of Basic Medical Sciences, Luohe Medical College, 148 Daxue Road, Luohe, Henan 462002, P.R. China

E-mail: wangfq22000@163.com

E-mail: songguohua54@163.com

Key words: miR-1291, mucin 1, esophageal squamous cell carcinoma, proliferation, invasion, apoptosis tases, deep tumor invasion and difficulty in early diagnosis, the majority of esophageal cancer patients have a relatively low survival rate $(2,3)$. Despite the many advances in surgery, chemotherapy and nutritional aid therapies at present, the long-term ESCC survival rate has only slightly improved in recent years (4). Therefore, it is a formidable task to study and explore novel biomarkers or therapeutic targets for ESCC patients. Recently some research findings have indicated that the initiation and development of ESCC involved the mutation of numerous oncogenes and anti-oncogenes, and thus were characterized by the synergetic effect of multiple genes, factors and steps $(5,6)$, yet more effort is still needed to study the genetic and molecular changes underlying the development of ESCC.

MicroRNAs (miRNAs) are small non-coding RNAs with 19-23 nucleotides $(7,8)$. Numerous studies have been performed on the role and related mechanism of miRNAs in numerous different kinds of diseases (9-12). miRNAs bind primarily to the 3'-untranslated region (3'UTR) of their target messenger RNAs (mRNAs) to reduce their stability and decrease the expression of target mRNAs at the post-transcriptional level (13), which play important roles in various biological processes including cell growth, proliferation, differentiation and death (14-19). Concerning chemotherapy in various types of cancers such as breast cancer, lung adenocarcinoma, glioblastoma, and ovarian cancer, recent studies have shown that miRNAs also act in important roles (20-24). In ESCC, altered expression of miR-1290, miR-655, miR-375 and others, has been observed (25-27), suggesting that miRNA deregulation plays an important role in ESCC development.

Recently, miR-1291 was found to be significantly downregulated in pancreatic and renal cell carcinomas, and restoration of miR-1291 function repressed tumorigenesis. To the best of our knowledge, there is no previous study concerning miR-1291 in ESCC biology. To gain insight into the potential mechanisms of miR-1291 in ESCC, we performed the relevant bioinformatic analyses using TargetScan and miRanda (28), and found that mucin 1 (MUC1) is a potential target of miR-1291. A previous study showed that the alteration of MUC1 is correlated with regional lymph node metastasis, and 
high-expression of MUC1 is associated with poor prognosis for esophageal cancer patients (29). Based on the above information, miR-1291 and MUC1 may play roles in the development of ESCC. The present study investigated miR-1291 and MUC1 expression levels in ESCC tissues from 54 patients, and evaluated the effect of miR-1291 upregulation on proliferation, invasion and apoptosis of ESCC cells, which provides new insight into the potential mechanisms of ESCC and identify a new possible target for early diagnosis or therapy.

\section{Materials and methods}

Clinical sample collection. Paired tumorous and adjacent nontumorous tissues were obtained from 54 patients with ESCC who underwent radical resection in the First Affiliated Hospital of Zhengzhou University and the First Affiliated Hospital of Luohe Medical College between 2012 and 2014 (Table I). Tissues were snap-frozen in liquid nitrogen and identified by pathological examinations after resection. None had received chemotherapy or radiotherapy before surgery. All patients consented to the use of their tissue samples in the present study. Human Research Ethics Committee of Zhengzhou University and Luohe Medical College approved the present study.

Cell lines and cell culture. Human ESCC EC9706 and EC-1 cell lines were purchased from the Type Culture Collection of the Chinese Academy of Sciences (Shanghai, China), were maintained in a RPMI-1640 medium containing $100 \mathrm{U} / \mathrm{ml}$ penicillin, $100 \mu \mathrm{l} / \mathrm{ml}$ streptomycin and $10 \%$ fetal bovine serum (FBS; Gibco-BRL, Gaithersburg, MD, USA) and incubated at $37^{\circ} \mathrm{C}$ and $5 \% \mathrm{CO}_{2}$.

RNA oligo-ribonucleotides and cell transfection. miR-1291 mimic and a negative control (NC) were chemically synthesized by Shanghai GenePharma Co. Ltd. The amount of miR-1291 mimic and $\mathrm{NC}$ is $7.92 \mu \mathrm{g} / 4 \times 10^{6}$ cells, respectively. Transfection was performed with a BTX ECM 2001 square wave electroporator (Genetronics Inc., San Diego, CA, USA) with electroporation settings adjusted according to the BTX ECM 2001 protocol. After transfection, EC9706 and EC-1 cells were seeded in 6 -well plates $\left(2 \times 10^{5}\right.$ cells/well). Cells from each cell line were subdivided into three groups: the non-transfected blank (blank), a mimic NC-transfected (NC) and the miR-1291 mimic-transfection groups (miR-1291). Cells were harvested for further experiments after $24-48 \mathrm{~h}$ post-transfection.

RNA extraction and quantitative real-time PCR. Relative levels of miR-1291 and MUC1 mRNA in ESCC tissue samples and adjacent non-tumorous tissue samples were determined by quantitative real-time PCR (qRT-PCR) assays. Total RNA was extracted with an RNA extraction kit (Qiagen, Venlo, The Netherlands), and RNA quality was confirmed using a NanoDrop 1000 spectrophotometer (Thermo Fisher Scientific, Wilmington, DE, USA). Reverse transcription was performed using MMLV RTase cDNA synthesis kit (Takara, Dalian, China). A cDNA library of miRNAs was constructed by QuantiMir cDNA kit (Takara). All protocols were performed according to the manufacturers' instructions, and qPCR was performed using the ABI Power SYBR-Green PCR Master Mix (Applied Biosystems, Foster City, CA, USA). The relative expression of miR-1291 was calculated using the comparative cycle threshold (CT; $2^{-\Delta \mathrm{Ct}}$ ) method with U6 snRNA as an endogenous control to normalize the data. $\beta$-actin was used as normalization for the relative levels of MUC1 mRNA. The calculation method was the same as presented.

Western blotting. Proteins were precipitated and determined by western blot analysis following the previously described procedures (26). Proteins were subjected to sodium dodecyl sulfate-polyacrylamide gel electrophoresis (SDS-PAGE) and transferred onto polyvinylidene difluoride (PVDF) membranes. After blocking, membranes were incubated overnight at $4^{\circ} \mathrm{C}$ with the diluted (1:500) primary antibody (polyclonal rabbit anti-MUC1; Santa Cruz). Following extensive washing, the membranes were incubated with the diluted $(1: 3,000)$ horseradish peroxidase-conjugated goat anti-rabbit IgG (Santa Cruz). Signals were detected using a chemiluminescence detection kit (Amersham Pharmacia Biotech, Piscataway, NJ, USA). An antibody against $\beta$-actin (Santa Cruz) served as an endogenous reference. Relative protein levels were calculated using $\beta$-actin as a loading control.

Cell Counting Kit-8 (CCK-8) assay. We used the CCK-8 assay (Dojindo Laboratories, Japan) according to the manufacturer's instructions to determine cell viability. Briefly, cells were seeded at a density of $2 \times 10^{3}$ cells/well in 96-well plates (in three replicate wells) and treated daily for four consecutive days with $10 \mu \mathrm{l} /$ well of CCK-8 solution. Optical density was measured at $450 \mathrm{~nm}$ to estimate the number of viable cells.

Colony formation assay. Twenty-four hours after RNA transfection, cells were suspended in $0.3 \%$ agarose with RPMI-1640 containing 10\% FBS and plated into four $6-\mathrm{cm}$ cell culture dishes on top of an existing layer of $0.6 \%$ agarose prepared with the same medium. The plates were incubated at $37^{\circ} \mathrm{C}$ in a $5 \% \mathrm{CO}_{2}$ incubator for 12 days. Colonies were stained with crystal violet, and those with $>50$ cells were scored as surviving colonies. The cloning efficiency was calculated by dividing the average number of colonies/dish by the number of plated cells.

Cell apoptosis detection. Flow cytometric assays were conducted using the Annexin V-FITC apoptosis detection kit I (BestBio, Shanghai, China), according to the manufacturer's instructions. Cells from the two groups (miR-1291 and NC) were harvested by trypsinization and resuspended at a density of $1 \times 10^{6}$ cells $/ \mathrm{ml}$ in $1 \mathrm{X}$ binding buffer. After double staining with propidium iodide (PI) and Annexin V-FITC, the samples were analyzed by flow cytometry and the data were analyzed using CellQuest software.

Transwell invasion assay. We assayed the invasion ability of cells using $6.5 \mathrm{~mm}$ diameter Transwell chambers with 8- $\mu \mathrm{m}$ membranes (Corning, USA). Twenty-four hours after post-transfection, EC9706 or EC-1 cells were added to the upper chambers, and the bottom wells were coated with $1 \mathrm{mg} / \mathrm{ml} \mathrm{Matrigel} \mathrm{for} \mathrm{the} \mathrm{invasion} \mathrm{assays,} \mathrm{while} \mathrm{a} \mathrm{medium}$ containing $10 \% \mathrm{FBS}$ was added to the lower chamber. After $24 \mathrm{~h}$ at $37^{\circ} \mathrm{C}$ in a $5 \% \mathrm{CO}_{2}$ humidified atmosphere, cells in the upper chamber were carefully scraped off using a cotton swab, 
Table I. Clinicopathological characteristics and the expression of miRNA-1291 and MUC1 mRNA of ESCC patients.

\begin{tabular}{|c|c|c|c|c|c|}
\hline Variables & $\mathrm{n}$ & $\begin{array}{l}\text { miRNA-1291 expression } \\
\quad(\text { median } \pm \text { SD })\end{array}$ & P-value & $\begin{array}{l}\text { Relative expression of } \\
\text { MUC1 mRNA } \\
(\text { median } \pm \text { SD) }\end{array}$ & P-value \\
\hline Gender & & & 0.511 & & 0.066 \\
\hline Male & 39 & $0.6159 \pm 0.32902$ & & $1.3410 \pm 0.20273$ & \\
\hline Female & 15 & $0.5507 \pm 0.31224$ & & $1.4527 \pm 0.17527$ & \\
\hline Age (years) & & & 0.080 & & 0.542 \\
\hline$\geq 60$ & 33 & $0.5364 \pm 0.25019$ & & $1.3855 \pm 0.16354$ & \\
\hline$<60$ & 21 & $0.6943 \pm 0.40011$ & & $1.3510 \pm 0.25058$ & \\
\hline Differentiation & & & 0.072 & & $0.028^{\mathrm{a}}$ \\
\hline Well & 15 & $0.5960 \pm 0.35603$ & & $1.2693 \pm 0.22008$ & \\
\hline Moderate & 31 & $0.6584 \pm 0.32421$ & & $1.3919 \pm 0.19580$ & \\
\hline Poor & 8 & $0.3663 \pm 0.08782$ & & $1.4875 \pm 0.05548$ & \\
\hline Tumor location & & & 0.519 & & $0.001^{\mathrm{a}}$ \\
\hline Low & 12 & $0.5442 \pm 0.35748$ & & $1.2108 \pm 0.21660$ & \\
\hline Middle & 42 & $0.6131 \pm 0.31525$ & & $1.4181 \pm 0.17178$ & \\
\hline TNM stage & & & $0.004^{\mathrm{a}}$ & & 0.232 \\
\hline I & 14 & $0.7586 \pm 0.45578$ & & $1.3207 \pm 0.26409$ & \\
\hline II & 29 & $0.6172 \pm 0.24326$ & & $1.3645 \pm 0.19642$ & \\
\hline III & 11 & $0.3418 \pm 0.08577$ & & $1.4573 \pm 0.04628$ & \\
\hline Nodal status & & & $<0.001^{\mathrm{a}}$ & & $0.002^{\mathrm{a}}$ \\
\hline Positive & 37 & $0.7014 \pm 0.33548$ & & $1.3159 \pm 0.21554$ & \\
\hline Negative & 17 & $0.3724 \pm 0.11519$ & & $1.4941 \pm 0.07272$ & \\
\hline
\end{tabular}

Statistical significance $\left({ }^{\mathrm{a}} \mathrm{P}<0.05\right)$. MUC1, mucin 1; ESCC, esophageal squamous cell carcinoma.

and cells that had migrated to the basal side of the membrane were fixed in methanol, stained with hematoxylin, mounted and dried at $80^{\circ} \mathrm{C}$ for $30 \mathrm{~min}$. The number of cells invading the Matrigel was counted in three randomly selected fields using an inverted microscope (magnification, x200). Each test was performed in triplicate.

Luciferase reporter assays. The wild-type MUC1 3'UTR fragments containing putative seed region for miR-1291 were amplified from the human genomic DNA by PCR. The mutagenesis of the seed region was achieved by overlap PCR. The wild-type (wt) MUC1 3'UTR and mutant (mut) MUC1 3'UTR fragments were inserted into the pmirGLO promoter vector (Promega) downstream of the luciferase gene to generate the recombinant luciferase reporter vectors pmirGLO-MUC1-wt and pmirGLO- MUC1-mut, respectively. For the luciferase reporter assay, the recombinant plasmids were co-transfected with miR-1291 mimic or NC into EC9706 cells. After 24 h, luciferase activity was measured using the Dual-Luciferase Reporter assay system (Promega) according to the manufacturer's instructions.

Statistical analysis. Statistical analyses were performed using SPSS 17.0 software. Data are expressed as mean \pm standard deviation (SD). The Student's t-test was performed to compare sample means. One-way analysis of variance (ANOVA) was used to analyze the significance between different samples.
$\mathrm{P}<0.05$ was considered to indicate a statistically significant result. Pearson's correlation analysis was used to analyze the correlation between different variable elements.

\section{Results}

Expression of miR-1291 and MUC1 mRNA in ESCC samples. We investigated the expression of miR-1291 and MUC1 in ESCC samples by qRT-PCR array. Compared with adjacent non-tumorous tissues, miR-1291 expression was significantly lower in ESCC tissues ( $\mathrm{P}<0.05$; Fig. 1B), while MUC1 mRNA showed the opposite trend $(\mathrm{P}<0.05$; Fig. 1A). These observations demonstrate that the expression of miR-1291 and MUC1 was negatively correlated in ESCC tissues. In addition, we further analyzed our data and found that miR-1291 expression level in ESCC tissues was associated with lymph node metastases and TNM stage $(\mathrm{P}<0.05$; Table I; Fig. $1 \mathrm{C}$ and $\mathrm{D})$. No significant differences were observed between miR-1291 expression and gender, age, differentiation or tumor location ( $\mathrm{P}>0.05$; Table I). Distinctly, the MUC1 mRNA expression level in ESCC tissues was associated with lymph node metastases, differentiation and tumor location $(\mathrm{P}<0.05$; Table I). There were no significant differences between MUC1 mRNA expression and TNM stage, gender or age $(\mathrm{P}>0.05$; Table I).

Upregulation of miR-1291 inhibits cell proliferation in EC9706 and EC-1 cells. The CCK-8 and colony formation 
A

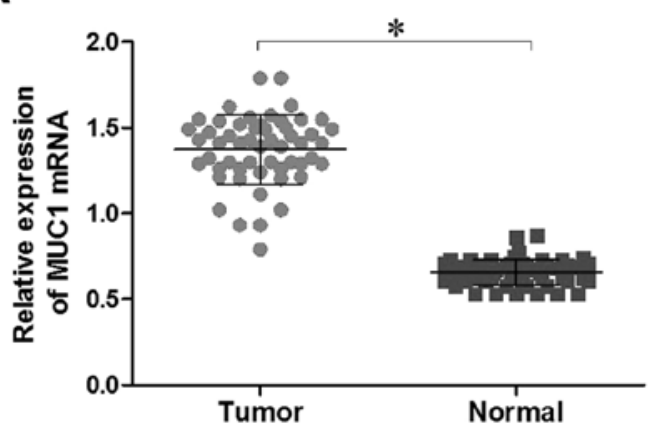

C

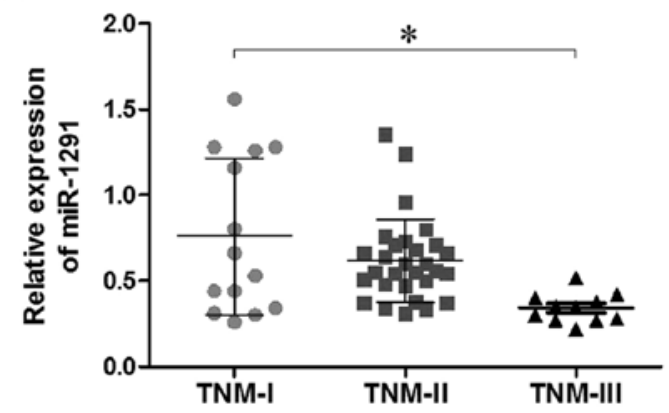

B

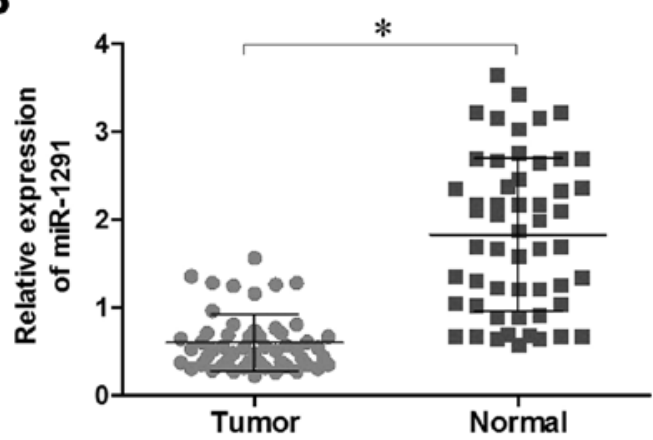

D

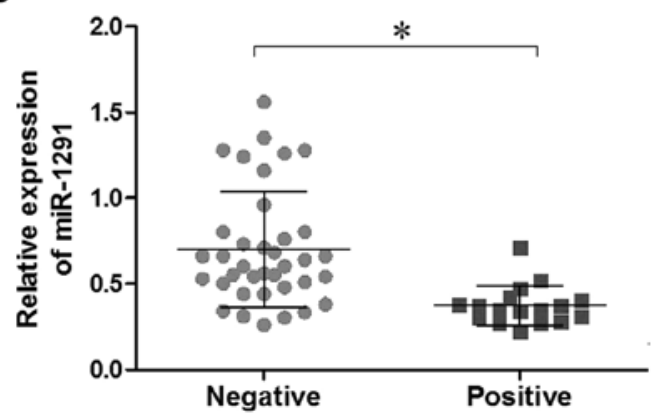

Figure 1. miR-1291 and MUC1 mRNA expression in ESCC and adjacent non-tumor tissues determined by quantitative real-time PCR (qRT-PCR). (A) MUC1 mRNA expression in ESCC tumor tissues was significantly higher than in normal tissues $(\mathrm{P}<0.05)$. (Tumor, ESCC tissues; and normal, adjacent non-tumor tissues). (B) Relative expression levels of miR-1291 in paired ESCC and adjacent non-tumor tissues $(\mathrm{P}<0.05)$. (C) The expression levels of miR-1291 were significantly lower in TNM-III than in TNM-I and TNM-II. (D) ESCC tissues were divided into two groups of metastasis-positive (positive) and metastasis-negative (negative) ESCC tissues. The low expression of miR-1291 was significantly associated with lymph node metastasis $(\mathrm{P}<0.05)$. Statistical significance $\left({ }^{*} \mathrm{P}<0.05\right)$. MUC1, mucin 1; ESCC, esophageal squamous cell carcinoma.

A

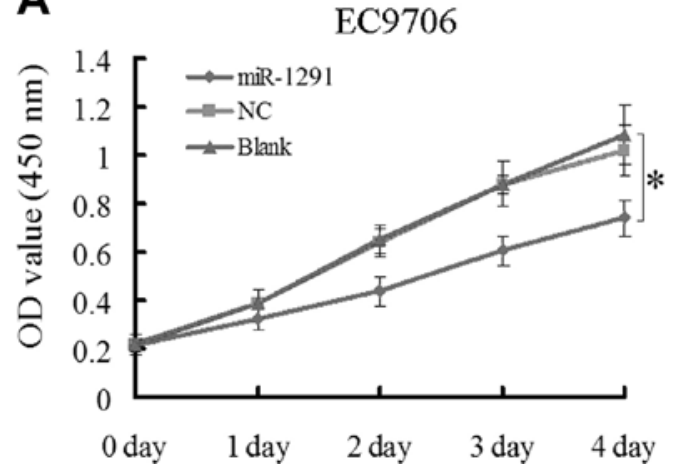

B

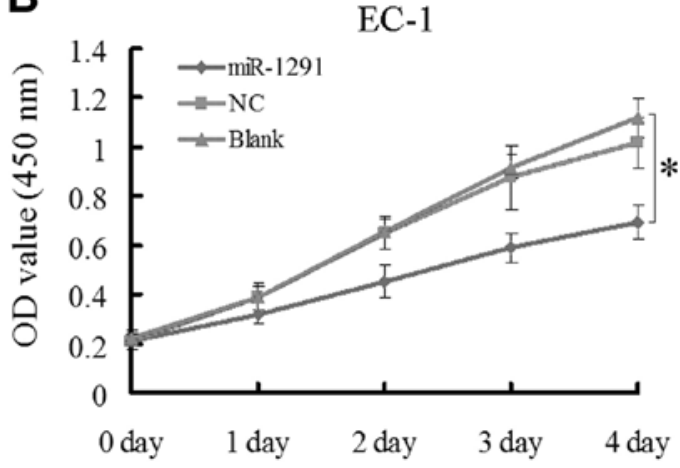

Figure 2. Overexpression of miR-1291 inhibits cell proliferation in ESCC cells. The proliferation of ESCC cell lines was determined by Cell Counting Kit-8 (CCK-8) assay after transfection with miR-1291 mimic or NC, proliferation of (A) EC9706 and (B) EC-1 cells in the miR-1291 group was significantly decreased compared with cells of the $\mathrm{NC}$ and blank groups after 2,3 and 4 days $(\mathrm{P}<0.05)$. Statistical significance $\left({ }^{*} \mathrm{P}<0.05\right)$. ESCC, esophageal squamous cell carcinoma; NC, negative control.

assays in EC9706 and EC-1 cell lines were performed to evaluate the effect of miR-1291 on cell growth. According to the CCK-8 assay results, the absorbance of EC9706 or EC-1 cells transfected with miR-1291 mimic was markedly decreased compared to the control groups (NC and blank control) from the second day onwards $(\mathrm{P}<0.05$; Fig. $2 \mathrm{~A}$ and $\mathrm{B})$. In the colony formation assay, the colony-forming activity of the miR-1291 mimic group was lower than that of the NC group in both EC9706 (Fig. 3A) and EC-1 (Fig. 3B) cells $(\mathrm{P}<0.05)$. Based on these results, we concluded that upregulation of miR-1291 inhibited the proliferation of EC9706 and EC-1 cells.
Upregulation of miR-1291 promotes apoptosis in EC9706 and $E C-1$ cells. In order to determine whether apoptosis resulted in proliferation inhibition, we performed a flow cytometric apoptosis assay. Our data showed that in contrast to NC groups the number of apoptotic EC9706 or EC-1 cells transfected with miR-1291 mimic increased significantly after $48 \mathrm{~h}(\mathrm{P}<0.05$; Fig. 4A and B). Based on these results, we concluded that upregulation of miR-1291 promotes apoptosis of EC9706 and EC-1 cells.

Upregulation of miR-1291 restricts cell invasion ability of EC9706 and EC-1 cells. A Transwell assay was performed 

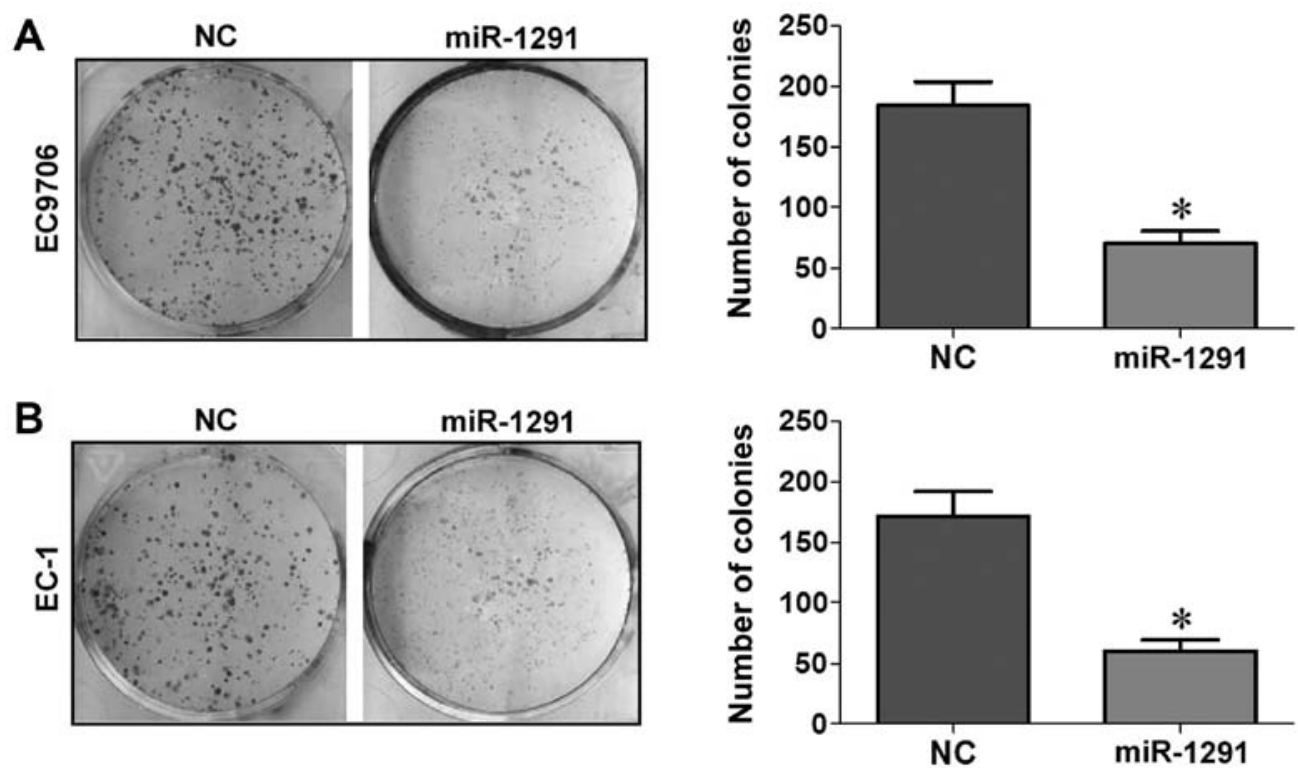

Figure 3. Overexpression of miR-1291 inhibits colony formation in ESCC cells. EC9706 and EC-1 cells were transfected with miR-1291 mimic or NC. Number of (A) EC9706 and (B) EC-1 colonies in the miR-1291 group was significantly reduced when compared to the NC groups, as measured by the colony-forming assay $(\mathrm{P}<0.05)$. Significance $\left({ }^{*} \mathrm{P}<0.05\right)$. ESCC, esophageal squamous cell carcinoma; NC, negative control.
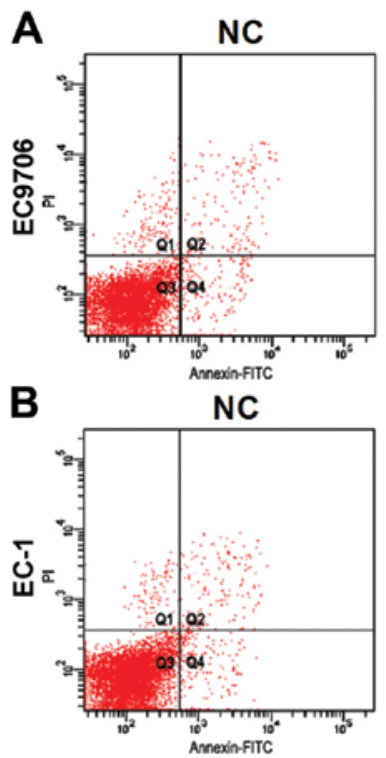

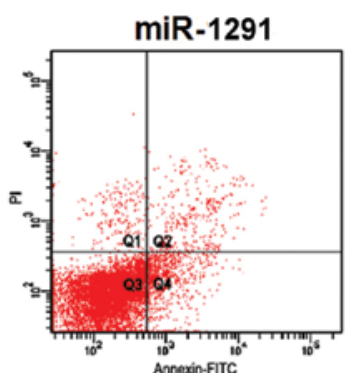

miR-1291

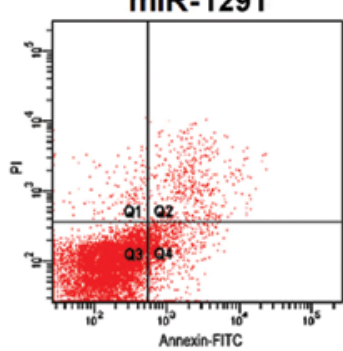

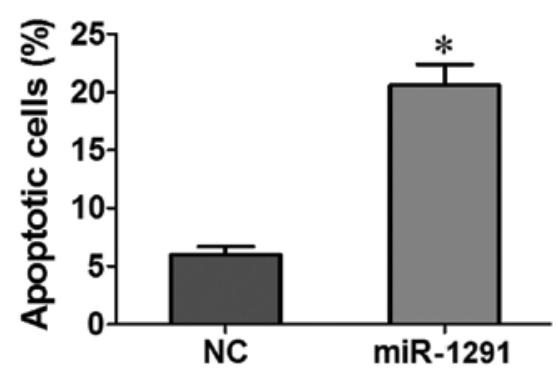

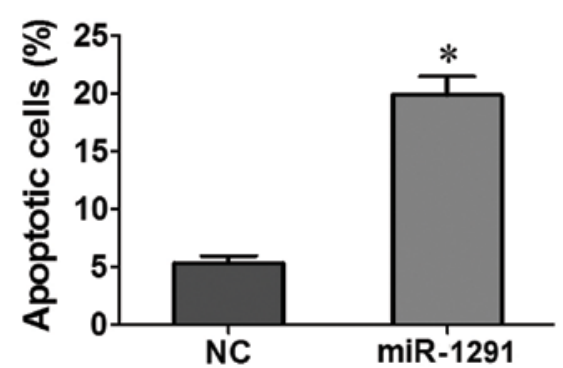

Figure 4. Overexpression of miR-1291 promotes apoptosis in ESCC cells. Flow cytometric apoptosis assays were used to assess the apoptosis effect of ESCC cells after transfection. (A) Statistically significant increases in the number of apoptotic cells in miR-1291 mimic-transfected EC9706 cells compared with that in the NC group $(\mathrm{P}<0.05)$. (B) Statistically significant increases of apoptotic cells in miR-1291 mimic-transfected EC-1 cells compared with that in the $\mathrm{NC}$ group $(\mathrm{P}<0.05)$. Statistical significance $(\mathrm{P}<0.05)$. ESCC, esophageal squamous cell carcinoma; NC, negative control

to evaluate the role of miR-1291 in regulating the invasion activity of EC9706 and EC-1 cells. Compared to the NC group, the number of EC9706 or EC-1 cells transfected with miR-1291 mimic that penetrated the Transwell membrane was significantly lower $(\mathrm{P}<0.05$; Fig. $5 \mathrm{~A}$ and $\mathrm{B})$. These results indicated that upregulation of miR-1291 restricted the invasion capacity of EC9706 or EC-1 cells.

miR-1291 downregulates MUC1 expression by binding to its 3'UTR. The TargetScan and miRanda prediction algorithms showed that the MUC1 3'UTR may be directly targeted by miR-1291 (Fig. 6A). In order to verify this targeting relationship, the wild-type and mutant human MUC1 3'UTR fragments were cloned downstream of the firefly luciferase reporter gene in pmirGLO vector (pmirGLO-MUC1-wt and pmirGLO-MUC1-mut) and then co-transfected with a miR-1291 mimic (or NC) into EC9706 cells. The relative luciferase activity of the reporter gene in EC9706 cells co-transfected with pmirGLO-MUC1-wt and miR-1291 mimic was significantly lower $(\mathrm{P}<0.05)$ compared with the control group (co-transfected pmirGLO-MUC1-wt and NC) (Fig. 6C). Among cells co-transfected with pmirGLO-MUC1-mut, 

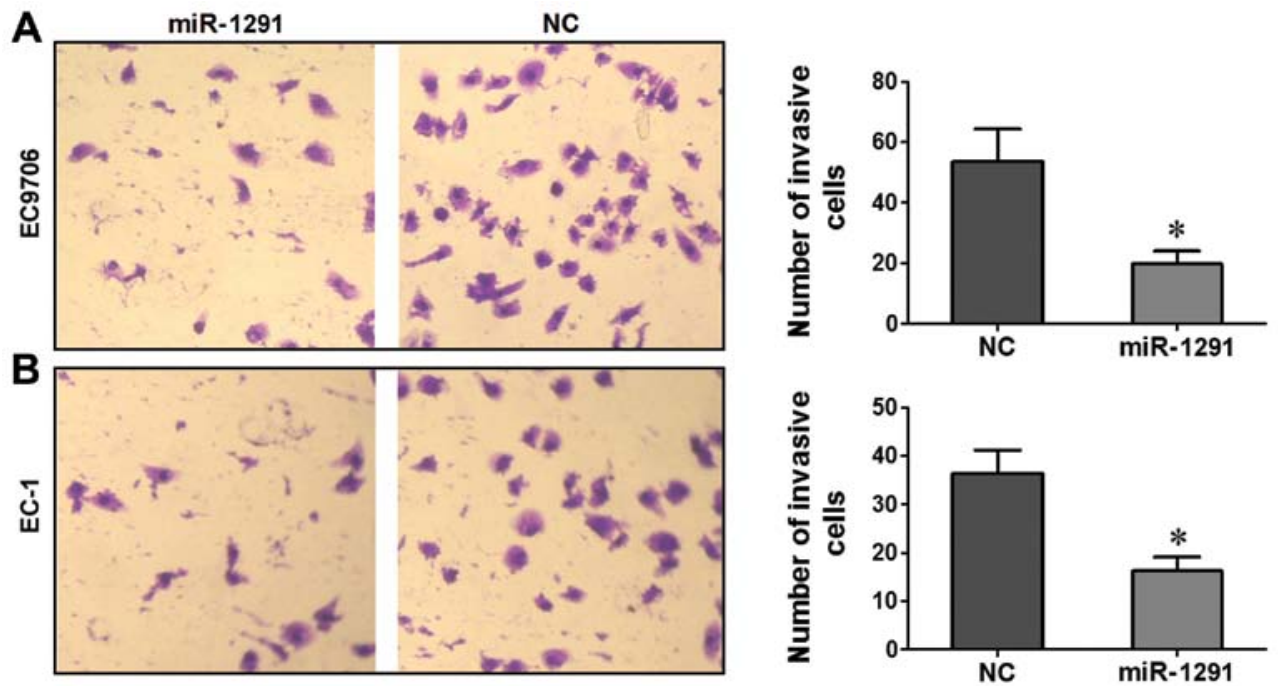

Figure 5. Overexpression of miR-1291 inhibits invasion in ESCC cells. Transwell assays were used to assess the invasive ability of ESCC cells after transfection. (A) Number of EC9706 cells of the miR-1291 group passing through the Matrigel was significantly lower than that in the NC group (P<0.05). (B) Number of EC-1 cells in the miR-1291 group passing through the Matrigel was significantly lower than that in the NC group (P<0.05). Statistical significance $($ " $\mathrm{P}<0.05)$. ESCC, esophageal squamous cell carcinoma; $\mathrm{NC}$, negative control.

A

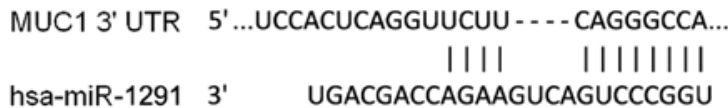

B

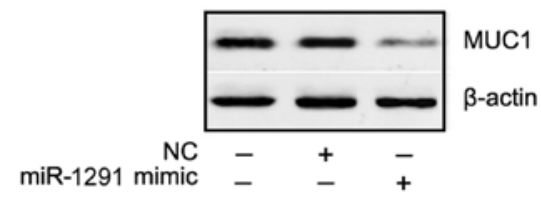

C

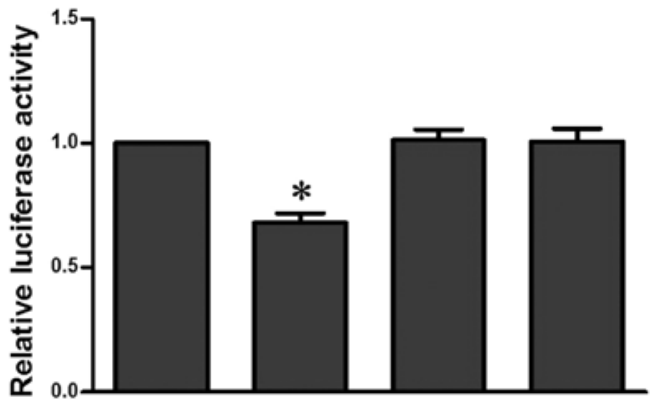

$\begin{array}{rllll}\text { NC } & + & - & + & - \\ \text { miR-1291 mimic } & - & + & - & + \\ \text { pmirGLO-MUC1-Wt 3'UTR } & + & + & - & - \\ \text { pmirGLO-MUC1-Mut 3'UTR } & - & - & + & +\end{array}$

Figure 6. MUC1 is a potential target of miR-1291. (A) The putative miR-1291 seed region for the MUC1 3'UTR. (B) Western blot analysis of MUC1 expression in transfected cells (EC9706). miR-1291 negatively regulated the expression of MUC1. $\beta$-actin was used as an endogenous reference. (C) Luciferase reporter assay. Luciferase reporter vectors containing wild-type or mutated 3'UTR of MUC1 were constructed and co-transfected into EC9706 cells with miR-1291 mimic or NC. There was no difference in luciferase activity between cells co-transfected with pmirGLO-MUC1-mut and miR-1291 mimic or NC. However, in cells co-transfected with pmirGLO-MUC1-wt, the relative luciferase activity of the cells co-transfected with miR1291 mimic was significantly lower than that of cells in the NC transfection group $(\mathrm{P}<0.05)$. Statistical significance ("P<0.05). MUC1, mucin $1 ; 3^{\prime} \mathrm{UTR}$, 3'-untranslated region.

no difference was found between the relative luciferase activity of cells co-transfected with miR-1291 mimic and cells co-transfected with NC. This result was confirmed by western blot analysis showing that MUC1 expression was downregulated in the EC9706 cells following transfection with miR-1291 mimic (Fig. 6B). These results indicated that miR-1291 negatively regulated MUC1 expression by directly binding to the 3'UTR seed region in ESCC. 
A

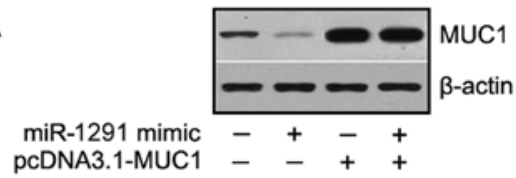

B

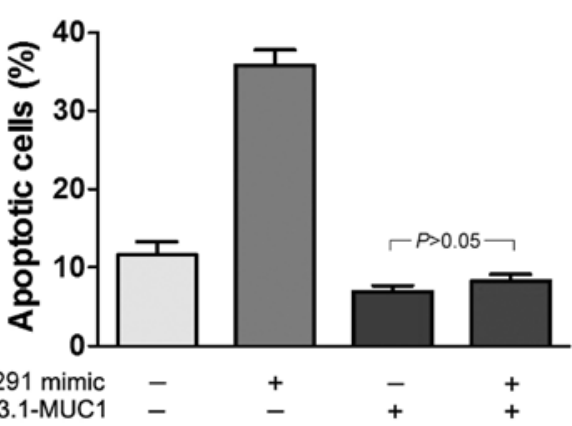

C

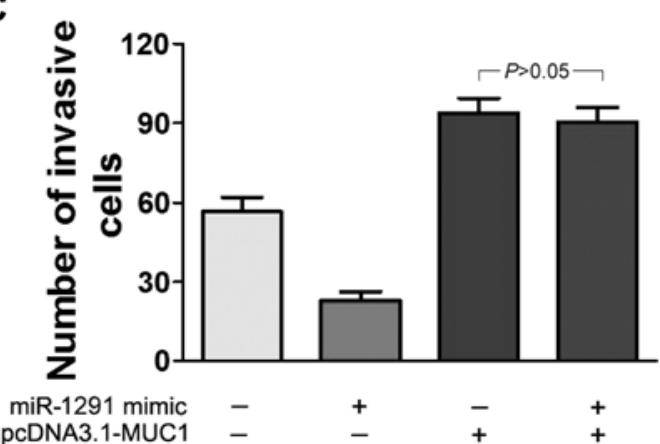

Figure 7. Expression of MUC1 abrogates the anti-migratory and proapoptosis functions of miR-1291. (A) Western blot analysis showed that co-transfection with pcDNA3.1-MUC1 (lacking the 3'UTR) and miR-1291 mimic increased MUC1 expression. $\beta$-actin was used as an endogenous reference. (B) Apoptosis assay showed that transfection with pcDNA3.1-MUC1 (lacking the 3'UTR) and miR-1291 mimic decreased the percentages of apoptotic cells $(\mathrm{P}<0.05)$. (C) Transfection with pcDNA3.1-MUC1 (lacking the $3^{\prime}$ UTR) and the miR-1291 mimic increased the average number of invading cells, as measured by the Transwell assay $(\mathrm{P}<0.05)$. MUC1, mucin 1; 3'UTR, 3'-untranslated region.

Expression of MUCl abrogates the anti-invasion and pro-apoptosis function of miR-1291. To further clarify MUC1 as a direct target of miR-1291, the function of MUC1 in miR-1291-mediated invasion and apoptosis was investigated. We constructed a recombinant expressing vector pcDNA3.1-MUC1, which contains MUC1 lacking the 3'UTR. The EC9706 cells were transfected with miR-1291 mimic or pcDNA3.1-MUC1 or co-transfected with both. Western blotting showed that co-transfection of miR-1291 mimic and pcDNA3.1-MUC1 led to an increase in MUC1 expression, and abrogated the effects of miR-1291 mimic (Fig. 7A). Furthermore, the apoptosis assay demonstrated that compared to cells only subjected to serum deprivation, the percentage of apoptotic cells transfected with miR-1291 mimic was significantly increased $(\mathrm{P}<0.05$; Fig. 7B). However, the percentage of apoptotic cells was significantly decreased in transfection with pcDNA3.1-MUC1 or co-transfection with pcDNA3.1-MUC1 and miR-1291 mimic. These data indicated that co-transfection with pcDNA3.1-MUC1 and miR-1291 mimic abrogated the effects of miR-1291 on cell apoptosis $(\mathrm{P}<0.05$; Fig. 7B). In the
Transwell assays, we found that co-transfection of pcDNA3.1MUC1 and miR-1291 mimic increased the average number of invading cells $(\mathrm{P}<0.05$; Fig. $7 \mathrm{C})$. Based on these results we further confirmed that 3'UTR of MUC1 is the action site of miR-1291.

\section{Discussion}

Increasing studies have shown that miRNAs play important roles in carcinogenesis and progression of esophagus cancer (EC). For instance, miR-1290 was significantly upregulated in ESCC tissue samples, and ectopic miR-1290 expression potently promoted ESCC cell growth, migration and invasion in vitro (25). miR-140 expression was decreased in the EC tissues and regulated the cell invasion of EC via controlling Slug expression (30). It has also been shown that miR-625 expression is lower in EC tissues than in the corresponding adjacent tissues and may regulate the proliferation and invasion of EC cells by targeting Sox 2 (31). miR-103/107 expression has been associated with poor survival rates for ESCC patients (32).

In the present study, miR-1291 expression was explored in ESCC. We analyzed the relationship between the miR-1291 expression levels and clinicopathological characteristics of ESCC. The results showed that miR-1291 was significantly downregulated in ESCC tissues and was correlated with lymph node metastases and TNM stage. These results indicated that miR-1291 may be a new diagnosis molecule or therapeutic target for ESCC. Moreover, cell biology experiments in EC9706 and EC-1 cells showed overexpression of miR-1291 inhibited proliferation, restricted cell invasion and resulted in an increased rate of cell apoptosis. These observations suggested that miR-1291 functioned as a tumor-suppressor in ESCC.

Bioinformatics analysis using TargetScan and miRanda revealed that MUC1 is one of the targets of miR-1291. MUC1 is a transmembrane protein that has been identified by its marked overexpression in human carcinomas $(33,34)$. Studies have shown that epithelial Muc1/MUC1 facilitates mucosal wound healing by enhancing cell migration and proliferation, protecting against apoptosis and mediating expression of mucosal modulators (35). In most epithelial-derived cancer cells, MUC1 is overexpressed and loses its apical polarity $(36,37)$. In various adenocarcinoma including ESCC, MUC1 overexpression is correlated with tumor growth, lymph node metastasis and resistance to apoptosis (38), involved in multiple cancer-associated pathways. MUC1 suppresses activation of the ARF-MDM2-p53 pathway (39). In MUC1expressing cells, a MUC1 co-operating NF- $\mathrm{KB}$ signaling pathway plays a critical role in cancer cell invasion (40). MUC1 deficiency impairs NFKB p65, Akt and MAPK pathways, which indicated that MUC1 appears to be a good therapeutic target to slow down esophageal tumor progression (41). In the present study, we detected MUC1 mRNA of tumorous and adjacent non-tumorous human esophagus tissues from 54 patients with ESCC. Our data showed that MUC1 mRNA levels were significantly increased in ESCC tissues and related to lymph node metastasis. In addition, we found that altered MUC1 expression levels were associated with ESCC differentiation status and tumor location. Our results clarified the 
relationship between the MUC1 expression levels and clinicopathological characteristics of ESCC.

To confirm that MUC1 was one of the direct functional targets of miR-1291, the 3'UTR region of MUC1 was amplified from human genomic DNA and inserted into the pmirGLO vector to construct a luciferase reporter plasmid, and qRT-PCR, western blotting, luciferase reporter and knockdown assays were performed. Further, restoration assays on invasion and apoptosis were performed and the results showed expression of MUC1 abrogates the anti-invasion and pro-apoptosis function of miR-1291, which confirmed that miR-1291 bound to the 3'UTR of MUC1 mRNA reduced stability and/or inhibited translation involved in proliferation, invasion and apoptosis of ESCC cells.

Collectively, our investigation identified significantly lower expression of miR-1291 in ESCC tissues and that overexpression of miR-1291 suppressed cell growth, invasion and promoted apoptosis in ESCC cells. Our results indicated that miR-1291 acts as a tumor suppressor by targeting MUC1 in ESCC. These new findings suggest that miR-1291 plays an important role in regulating carcinogenesis and development of ESCC and is of value to the diagnosis and therapy of ESCC.

\section{Acknowledgements}

The present study was supported by the Luohe Medical College (no. 2014-S-LMC23). We would also like to thank Alison Beamish at the University of British Columbia for her assistance with English language and grammatical editing of the manuscript.

\section{References}

1. Napier KJ, Scheerer M and Misra S: Esophageal cancer: A Review of epidemiology, pathogenesis, staging workup and treatment modalities. World J Gastrointest Oncol 6: 112-120, 2014.

2. Kunisaki C, Makino H, Kimura J, Oshima T, Fujii S, Takagawa R, Kosaka T, Ono HA and Akiyama H: Impact of lymph-node metastasis site in patients with thoracic esophageal cancer. J Surg Oncol 101: 36-42, 2010.

3. Rice TW, Rusch VW, Apperson-Hansen C, Allen MS, Chen LQ, Hunter JG, Kesler KA, Law S, Lerut TE, Reed CE, et al: Worldwide esophageal cancer collaboration. Dis Esophagus 22: $1-8,2009$.

4. Mizoguchi K, Ishiguro H, Kimura M, Takahashi H, Sakamoto N, Tanaka $\mathrm{T}$ and Takeyama H: Induction of apoptosis by eicosapentaenoic acid in esophageal squamous cell carcinoma. Anticancer Res 34: 7145-7149, 2014.

5. Zhu X, Ding M, Yu ML, Feng MX, Tan LJ and Zhao FK: Identification of galectin-7 as a potential biomarker for esophageal squamous cell carcinoma by proteomic analysis. BMC Cancer 10: 290, 2010.

6. Shigeoka M, Urakawa N, Nishio M, Takase N, Utsunomiya S, Akiyama H, Kakeji Y,Komori T, Koma Y and Yokozaki H: Cyr61 promotes CD204 expression and the migration of macrophages via MEK/ERK pathway in esophageal squamous cell carcinoma Cancer Med 4: 437-446, 2015.

7. Ambros V: The functions of animal microRNAs. Nature 431: 350-355, 2004.

8. Hobert O: Gene regulation by transcription factors and microRNAs. Science 319: 1785-1786, 2008.

9. Schaefer JS, Attumi T, Opekun AR, Abraham B, Hou J, Shelby H, Graham DY, Streckfus C and Klein JR: MicroRNA signatures differentiate Crohn's disease from ulcerative colitis. BMC Immunol 16: 5, 2015.

10. Hoss AG, Labadorf A, Latourelle JC, Kartha VK, Hadzi TC, Gusella JF, MacDonald ME, Chen JF, Akbarian S, Weng Z, et al: miR-10b-5p expression in Huntington's disease brain relates to age of onset and the extent of striatal involvement. BMC Med Genomics 8: 10, 2015.

11. Trionfini P, Benigni A and Remuzzi G: MicroRNAs in kidney physiology and disease. Nat Rev Nephrol 11: 23-33, 2015.
12. Gupta S and Li L: Modulation of miRNAs in pulmonary hypertension. Int J Hypertens 2015: 169069, 2015.

13. Bartel DP: MicroRNAs: Target recognition and regulatory functions. Cell 136: 215-233, 2009

14. Kong W, He L, Richards EJ, Challa S, Xu CX, Permuth-Wey J, Lancaster JM, Coppola D, Sellers TA, Djeu JY, et al: Upregulation of miRNA-155 promotes tumour angiogenesis by targeting VHL and is associated with poor prognosis and triple-negative breast cancer. Oncogene 33: 679-689, 2014.

15. Yang YK,XiWY,Xi RX,Li JY,Li Q and Gao YE: MicroRNA-494 promotes cervical cancer proliferation through the regulation of PTEN. Oncol Rep 33: 2393-2401, 2015.

16. Zhang $\mathrm{Y}$, Han $\mathrm{T}$, Wei $\mathrm{G}$ and Wang $\mathrm{Y}$ : Inhibition of microRNA$17 / 20$ a suppresses cell proliferation in gastric cancer by modulating UBE2C expression. Oncol Rep 33: 2529-2536, 2015.

17. Cao Q, Dong P, Wang Y, Zhang J, Shi X and Wang Y: miR-218 suppresses cardiac myxoma proliferation by targeting myocyte enhancer factor 2D. Oncol Rep 33: 2606-2612, 2015.

18. Hwang HW and Mendell JT: MicroRNAs in cell proliferation, cell death, and tumorigenesis. Br J Cancer 96 (Suppl): R40-R44, 2007.

19. Wang Z, Ma X, Cai Q, Wang X, Yu B, Cai Q, liu B, Zhu Z and Li C: MiR-199a-3p promotes gastric cancer progression by targeting ZHX1. FEBS Lett 588: 4504-4512, 2014.

20. Yao YS, Qiu WS, Yao RY, Zhang Q, Zhuang LK, Zhou F, Sun LB and Yue L: miR-141 confers docetaxel chemoresistance of breast cancer cells via regulation of EIF4E expression. Oncol Rep 33: 2504-2512, 2015

21. Hu H, Li S, Cui X, Lv X, Jiao Y, Yu F, Yao H, Song E, Chen Y, Wang M, et al: The overexpression of hypomethylated miR-663 induces chemotherapy resistance in human breast cancer cells by targeting heparin sulfate proteoglycan 2 (HSPG2). J Biol Chem 288: 10973-10985, 2013.

22. Feng B, Wang R, Song HZ and Chen LB: MicroRNA-200b reverses chemoresistance of docetaxel-resistant human lung adenocarcinoma cells by targeting E2F3. Cancer 118: 3365-3376, 2012.

23. Lee D, Sun S, Zhang XQ, Zhang PD, Ho AS, Kiang KM, Fung CF, Lui WM and Leung GK: MicroRNA-210 and endoplasmic reticulum chaperones in the regulation of chemoresistance in glioblastoma. J Cancer 6: 227-232, 2015.

24. Zhou Y, Wang M, Wu J, Jie Z, Chang S and Shuang T: The clinicopathological significance of miR-1307 in chemotherapy resistant epithelial ovarian cancer. J Ovarian Res 8: 23, 2015.

25. Li M, He XY, Zhang ZM, Li S, Ren LH, Cao RS, Feng YD, Ji YL, Zhao Y and Shi RH: MicroRNA-1290 promotes esophageal squamous cell carcinoma cell proliferation and metastasis. World J Gastroenterol 21: 3245-3255, 2015.

26. Wang Y, Zang W, Du Y, Ma Y, Li M, Li P, Chen X, Wang T, Dong Z and Zhao G: Mir-655 up-regulation suppresses cell invasion by targeting pituitary tumor-transforming gene-1 in esophageal squamous cell carcinoma. J Transl Med 11: 301, 2013.

27. Isozaki Y, Hoshino I, Akutsu Y, Hanari N, Mori M, Nishimori T, Murakami K, Akanuma N, Takeshita N, Maruyama T, et al: Usefulness of microRNA 375 as a prognostic and therapeutic tool in esophageal squamous cell carcinoma. Int J Oncol 46: 1059-1066, 2015.

28. John B, Enright AJ, Aravin A, Tuschl T, Sander C and Marks DS: Human microRNA targets. PLoS Biol 2: e363, 2004.

29. Song ZB, Gao SS, Yi XN, Li YJ, Wang QM, Zhuang ZH and Wang LD: Expression of MUC1 in esophageal squamous-cell carcinoma and its relationship with prognosis of patients from Linzhou city, a high incidence area of northern China. World J Gastroenterol 9: 404-407, 2003.

30. Li W, Jiang G, Zhou J, Wang H, Gong Z, Zhang Z, Min K, Zhu H and Tan Y: Down-regulation of miR-140 induces EMT and promotes invasion by targeting Slug in esophageal cancer. Cell Physiol Biochem 34: 1466-1476, 2014.

31. Wang Z, Qiao Q, Chen M, Li X, Wang Z, Liu C and Xie Z: miR-625 down-regulation promotes proliferation and invasion in esophageal cancer by targeting Sox2. FEBS Lett 588: 915-921, 2014.

32. Guo Y, Chen Z, Zhang L, Zhou F, Shi S, Feng X, Li B, Meng X, Ma X, Luo M, et al: Distinctive microRNA profiles relating to patient survival in esophageal squamous cell carcinoma. Cancer Res 68: 26-33, 2008.

33. Kufe DW: Functional targeting of the MUC1 oncogene in human cancers. Cancer Biol Ther 8: 1197-1203, 2009.

34. Kufe DW: Mucins in cancer: Function, prognosis and therapy. Nat Rev Cancer 9: 874-885, 2009. 
35. Banerjee D, Fernandez HR, Patil PB, Premaratne P, QuidingJärbrink M and Lindén SK: Epithelial MUC1 promotes cell migration, reduces apoptosis and affects levels of mucosal modulators during acetylsalicylic acid (aspirin)-induced gastropathy. Biochem J 465: 423-431, 2015.

36. Singh PK and Hollingsworth MA: Cell surface-associated mucins in signal transduction. Trends Cell Biol 16: 467-476, 2006.

37. Mather IH, Jack LJ, Madara PJ and Johnson VG: The distribution of MUC1, an apical membrane glycoprotein, in mammary epithelial cells at the resolution of the electron microscope: Implications for the mechanism of milk secretion. Cell Tissue Res 304: 91-101, 2001.

38. Nath S and Mukherjee P: MUC1: A multifaceted oncoprotein with a key role in cancer progression. Trends Mol Med 20: 332-342, 2014
39. Raina D, Ahmad R, Chen D, Kumar S, Kharbanda S and Kufe D: MUC1 oncoprotein suppresses activation of the ARF-MDM2-p53 pathway. Cancer Biol Ther 7: 1959-1967, 2008.

40. Mori Y, Akita K, Tanida S, Ishida A, Toda M, Inoue M, Yashiro M, Sawada T, Hirakawa K and Nakada H: MUC1 protein induces urokinase-type plasminogen activator (uPA) by forming a complex with NF- $\kappa \mathrm{B}$ p65 transcription factor and binding to the uPA promoter, leading to enhanced invasiveness of cancer cells. J Biol Chem 289: 35193-35204, 2014.

41. Gronnier C, Bruyère E, Lahdaoui F, Jonckheere N, Perrais M, Leteurtre E, Piessen G, Mariette C and Van Seuningen I: The MUC1 mucin regulates the tumorigenic properties of human esophageal adenocarcinomatous cells. Biochim Biophys Acta 1843: 2432-2437, 2014. 